\title{
Kinematic Comparison of Exponential and Cayley Maps in the Planar Motion Group
}

\author{
İlhan Karakılıç* and Soner Erkuş \\ (Communicated by Kazım İlarslan)
}

\begin{abstract}
In this work, the exponential and the Cayley maps, from the Lie algebra $\mathfrak{s e}(2)$ of the planar motion group $S E(2)$, to the group itself are studied. The comparison between these maps on $S E(2)$ is given by using the Rodrigues vector. A three joint mechanism is discussed as an application.
\end{abstract}

Keywords: Kinematics; Lie group; planar motion group; exponential map; cayley map; Rodrigues vector.

AMS Subject Classification (2020): Primary: 53A17.

\section{Introduction}

Technological advancements force us to use computers in many fields. The usage of matrices in computer sciences is extremely important, since the mathematical algorithms in computer programmings are widely done by matrices. However the classical kinematic works are oftenly 'handmade' procedures. In simple terms the steps are; finding proper equations to a given problem, derivations of these equations, and obtain solutions to the problem. It is natural to use computer programs in these steps. 'Lie Group' and 'Lie Algebra' structures use direct matrix methods. If we introduce these structures to kinematics, computer methods may be applicaple for kinematic problems.

In this sense, the comparison between Cayley and Exponential maps in planar motion is given in terms of a well known subject, the Rodrigues vector $[2,6,14]$. The maps are defined from se(2) to SE(2). As it is known, Rodrigues vector characterizes the spatial motion (it is the direction of axis of rotation at that moment). For this reason planar motion group is examined by means of Rodrigues vector by extanding the plane to the Euclidean three space.

Some applications of Lie groups and Lie algebras to robotics are discussed in the following references. Selig's lecture notes [11] contains the basic knowledge from Chasles's theorem, lower Reuleaux's pairs to the Rodrigues formula. For the usage of Lie groups to the large rotations, it is worth to see [1]. The relation between the derivatives in Lie algebra and classical centrode curves of a rigid body motion is established in [8] and point-plane constraints are studied in [12]. The Lie group theory applications in computer science is constituted in [5,9]. One can examine the configuration manifolds for mobile robots $[13,15,16]$, for the extensive usage of this context. Cayley transformation is well known in $S O(3)$, and it is generalized to $S E(3)$ by Selig. He expressed the comparison between the Cayley map and the exponential map in $S E(3)$ [7].

In the view of Selig's work [7] we investigated similar decisions in the planar motion group $S E(2)$ and a new result, equation (4.7), is derived. In the last section, as an application, the length of Rodrigues vector and the position of end effector in a three joint mechanism are graphically signified.

\section{The Matrix Lie Group in the Plane Kinematics}

Planar motion group $S E(2)$ is the matrix Lie group in the plane kinematics. It can be represented as, 


$$
S=\left(\begin{array}{ccc}
\cos \theta & -\sin \theta & t_{x} \\
\sin \theta & \cos \theta & t_{y} \\
0 & 0 & 1
\end{array}\right)=\left(\begin{array}{cc}
R & t \\
0 & 1
\end{array}\right) .
$$

where $R$ is a rotation matrix and $t$ is translation vector in Euclidean space $\mathbb{E}^{2}$. Here $S$ defines a general rigid transformation, which is a rotation about a fixed point, the center of rotation and a traslation along a vector $\vec{t}=\left(t_{x}, t_{y}\right)^{T}$.

If $\vec{q}=\left(q_{x}, q_{y}\right)^{T}$, is the center of rotation, then rotation about this point is given by the conjugation,

$$
\begin{aligned}
S & =\left(\begin{array}{cc}
I_{2} & q \\
0 & 1
\end{array}\right)\left(\begin{array}{cc}
R & 0 \\
0 & 1
\end{array}\right)\left(\begin{array}{cc}
I_{2} & -q \\
0 & 1
\end{array}\right) \\
& =\left(\begin{array}{cc}
R & \left(I_{2}-R\right) q \\
0 & 1
\end{array}\right) \\
& =\left(\begin{array}{ccc}
\cos \theta & -\sin \theta & q_{y} \sin \theta+q_{x}(1-\cos \theta) \\
\sin \theta & \cos \theta & q_{y}(1-\cos \theta)-q_{x} \sin \theta \\
0 & 0 & 1
\end{array}\right)=\left(\begin{array}{cc}
R & t \\
0 & 1
\end{array}\right),
\end{aligned}
$$

where $I_{2}$ is the $2 \times 2$ identity matrix. Thus,

$$
\left(I_{2}-R\right) \vec{q}=\vec{t},
$$

yields the fixed point or center of rotation $q$, where we avoid taking a pure translation, i.e. $R \neq I_{2}$ (for details see $[8,9])$.

\section{The Exponential and The Cayley Maps}

Let $\mathrm{G}$ be a matrix Lie group and $\mathfrak{g}$ be its Lie algebra.

Definition 3.1. The exponential map, $\exp : \mathfrak{g} \rightarrow G$, can be defined by the ordinary series;

$$
e^{\Omega}=\sum_{k=0}^{\infty} \frac{\Omega^{k}}{k !}
$$

where $\Omega$ is an $n \times n$ matrix.

Consider the exponential map on rotation group $S O(2)$, which is the set of $2 \times 2$ real orthogonal matrices with determinant +1 . Its Lie algebra $\mathfrak{s o}(2)$ consists of $2 \times 2$ real skew-symmetric matrices. Let

$$
\Omega=\left(\begin{array}{cc}
0 & -\theta \\
\theta & 0
\end{array}\right)
$$

be an element of $\mathfrak{s o}(2)$. We need to find inductive formula expressing the powers $\Omega^{n}$. If

$$
\left(\begin{array}{cc}
0 & -\theta \\
\theta & 0
\end{array}\right)=\theta\left(\begin{array}{cc}
0 & -1 \\
1 & 0
\end{array}\right)
$$

then

$$
\begin{aligned}
& \Omega^{2}=-\theta^{2} I_{2}, \\
& \Omega^{3}=-\theta^{3} J_{2}, \\
& \Omega^{4}=\theta^{4} I_{2},
\end{aligned}
$$

where

$$
J_{2}=\left(\begin{array}{cc}
0 & -1 \\
1 & 0
\end{array}\right)
$$


Therefore,

$$
\begin{aligned}
\Omega^{4 n} & =\theta^{4 n} I_{2}, \\
\Omega^{4 n+1} & =\theta^{4 n+1} J_{2}, \\
\Omega^{4 n+2} & =-\theta^{4 n+2} I_{2}, \\
\Omega^{4 n+3} & =-\theta^{4 n+3} J_{2} .
\end{aligned}
$$

So one can obtain the Lie group element,

$$
\begin{aligned}
e^{\Omega} & =I_{2}+\frac{\theta}{1 !} J_{2}-\frac{\theta^{2}}{2 !} I_{2}-\frac{\theta^{3}}{3 !} J_{2}+\frac{\theta^{4}}{4 !} I_{2}+\frac{\theta^{5}}{5 !} J_{2}-\frac{\theta^{6}}{6 !} I_{2}-\frac{\theta^{7}}{7 !} J_{2} \ldots \\
& =\left(1-\frac{\theta^{2}}{2 !}+\frac{\theta^{4}}{4 !}-\frac{\theta^{6}}{6 !}+\ldots\right) I_{2}+\left(\frac{\theta}{1 !}-\frac{\theta^{3}}{3 !}+\frac{\theta^{5}}{5 !}-\frac{\theta^{7}}{7 !}+\ldots\right) J_{2} \\
& =\cos \theta I_{2}+\sin \theta J_{2}=\left(\begin{array}{cc}
\cos \theta & -\sin \theta \\
\sin \theta & \cos \theta
\end{array}\right)
\end{aligned}
$$

$e^{\Omega}$ is an orthogonal matrix of determinant +1 , which defines rotation as it is expected.

To investigate the exponential map on $S E(2)$ we have to find the Lie algebra element close to the identity by differentiating the Lie group element $S$ at $\theta=0$. Hence

$$
\left.\frac{d}{d \theta} S\right|_{\theta=0}=K
$$

or

$$
\begin{aligned}
K & =\left.\frac{d}{d \theta}\left(\begin{array}{ccc}
\cos \theta & -\sin \theta & q_{y} \sin \theta+q_{x}(1-\cos \theta) \\
\sin \theta & \cos \theta & q_{y}(1-\cos \theta)-q_{x} \sin \theta \\
0 & 0
\end{array}\right)\right|_{\theta=0} \\
& =\left(\begin{array}{ccc}
0 & -1 & q_{y} \\
1 & 0 & -q_{x} \\
0 & 0 & 0
\end{array}\right)=\left(\begin{array}{cc}
J_{2} & -J_{2} q \\
0 & 0
\end{array}\right)
\end{aligned}
$$

where $K$ satisfies the following cubic equation;

$$
K^{3}+K=0
$$

A general element of the Lie algebra can be written as $\theta K$ (see [9]). Thus

$$
\begin{aligned}
e^{\theta K} & =I_{3}+\frac{\theta}{1 !} K+\frac{\theta^{2}}{2 !} K^{2}-\frac{\theta^{3}}{3 !} K-\frac{\theta^{4}}{4 !} K^{2}+\frac{\theta^{5}}{5 !} K+\frac{\theta^{6}}{6 !} K^{2}-\frac{\theta^{7}}{7 !} K \ldots \\
& =I_{3}+\left(\frac{\theta}{1 !}-\frac{\theta^{3}}{3 !}+\frac{\theta^{5}}{5 !}-\frac{\theta^{7}}{7 !}+\ldots\right) K+\left(1-1+\frac{\theta^{2}}{2 !}-\frac{\theta^{4}}{4 !}+\frac{\theta^{6}}{6 !}-\ldots\right) K^{2} \\
& =I_{3}+\sin \theta K+(1-\cos \theta) K^{2},
\end{aligned}
$$

where $I_{3}$ is the $3 \times 3$ identity matrix and this formula obeys the familiar form of the Rodrigues formula in $S O(3)$. Hence we obtain,

$$
e^{\theta K}=\left(\begin{array}{ccc}
\cos \theta & -\sin \theta & q_{y} \sin \theta+q_{x}(1-\cos \theta) \\
\sin \theta & \cos \theta & q_{y}(1-\cos \theta)-q_{x} \sin \theta \\
0 & 0 & 1
\end{array}\right)
$$

Similar to the exponential map, there exists a rational polynomial map from the skew-symmetric matrices to the orthogonal matrices, which is known as the Cayley map.

Definition 3.2. Let $A \in \mathfrak{g}$ be an $n \times n$ skew-symmetric matrix then the map, Cay: $\mathfrak{g} \rightarrow G$, to the group of orthogonal matrices is called the Cayley map, where

$$
\operatorname{Cay}(A)=\left(I_{n}+A\right)\left(I_{n}-A\right)^{-1},
$$

and $I_{n}$ is an $n \times n$ identity matrix. 
Let us define a skew symmetric matrix A as,

$$
A=\left(\begin{array}{cc}
0 & -a_{12} \\
a_{12} & 0
\end{array}\right)
$$

then

$$
\operatorname{Cay}(A)=\left(I_{2}+A\right)\left(I_{2}-A\right)^{-1},
$$

and the inverse of $\left(I_{2}-A\right)$ can be written as

$$
\left(I_{2}-A\right)^{-1}=I_{2}+A+A^{2}+A^{3}+\ldots,
$$

(3.4) converges in some neighborhood of $A=0$. Since $A$ satisfies its charachteristic equation,

$$
A^{2}+a_{12}^{2} I_{2}=0
$$

From (3.4) and (3.5) we have

$$
\left(I_{2}-A\right)^{-1}=\frac{1}{1+a_{12}^{2}}\left(I_{2}+A\right) .
$$

Therefore

$$
\begin{aligned}
\operatorname{Cay}(A) & =\left(I_{2}+A\right)\left(I_{2}+A\right) \frac{1}{1+a_{12}^{2}} \\
& =\left(\frac{1-a_{12}^{2}}{1+a_{12}^{2}}\right) I_{2}+\left(\frac{2}{1+a_{12}^{2}}\right) A .
\end{aligned}
$$

The result (3.6) in $S O(2)$ can be extended to the group of planar motion $S E(2)$. Let $M$ be an element of $S E(2)$;

$$
M=\left(\begin{array}{cc}
\Re & u \\
0 & 1
\end{array}\right),
$$

where $\Re$ is a $2 \times 2$ rotation matrix and $u=\left(u_{1}, u_{2}\right)^{T}$ is a translation vector in $\mathbb{E}^{2}$. On the other hand a Lie algebra element can be written as,

$$
m=\left(\begin{array}{cc}
A & v \\
0 & 0
\end{array}\right),
$$

where $A$ is an $2 \times 2$ skew-symmetric matrix and $v=\left(v_{1}, v_{2}\right)^{T}$ is a velocity vector. Cayley map is originally defined on skew-symmetric matrices. Hovewer using formula (3.3) one can extend Cayley map to $S E(2)$, by

$$
\operatorname{Cay}_{3}(m)=\left(I_{3}+m\right)\left(I_{3}-m\right)^{-1},
$$

subscript 3 denotes the $2 \times 2$ representation of $S E(2)$. $m$ satisfies its charachteritic equation,

$$
m^{3}+a_{12}^{2} m=0,
$$

This leads to the formula,

$$
\begin{aligned}
\operatorname{Cay}_{3}(m) & =\left(I_{3}+m\right)\left(I_{3}-m\right)^{-1} \\
& =I_{3}+\frac{2}{1+a_{12}^{2}}\left(m+m^{2}\right) \\
& =\left(\begin{array}{ccc}
1-\frac{2 a_{12}^{2}}{1+a_{12}^{2}} & -\frac{2 a_{12}}{1+a_{12}^{2}} & \frac{v_{1}-a_{12} v_{2}}{1+a_{12}^{2}} \\
\frac{2 a_{12}}{1+a_{12}^{2}} & 1-\frac{2 a_{12}^{2}}{1+a_{12}^{2}} & \frac{a_{12} v_{1}+v_{2}}{1+a_{12}^{2}} \\
0 & 0 & 1
\end{array}\right) .
\end{aligned}
$$




\section{Comparison Between The Exponential Map and The Cayley Map}

The equivalance of the range spaces of Cayley and exponential maps motivates us to compare them. Therefore $e^{\theta K}=\mathrm{Cay}_{3}(m)$. This implies

$$
\left(\begin{array}{ccc}
\cos \theta & -\sin \theta & q_{y} \sin \theta+q_{x}(1-\cos \theta) \\
\sin \theta & \cos \theta & q_{y}(1-\cos \theta)-q_{x} \sin \theta \\
0 & 0 & 1
\end{array}\right)=\left(\begin{array}{ccc}
1-\frac{2 a_{12}^{2}}{1+a_{12}^{2}} & -\frac{2 a_{12}}{1+a_{12}^{2}} & \frac{v_{1}-a_{12} v_{2}}{1+a_{12}^{2}} \\
\frac{2 a_{12}}{1+a_{12}^{2}} & 1-\frac{2 a_{12}^{2}}{1+a_{12}^{2}} & \frac{a_{12} v_{1}+v_{2}}{1+a_{12}^{2}} \\
0 & 0 & 1
\end{array}\right) .
$$

Hence from the rotation parts of the matrices;

$$
\begin{aligned}
1-\frac{2 a_{12}^{2}}{1+a_{12}^{2}} & =\cos \theta \\
\frac{2 a_{12}}{1+a_{12}^{2}} & =\sin \theta .
\end{aligned}
$$

Thus,

$$
a_{12}=\tan \left(\frac{\theta}{2}\right) .
$$

As a result we obtain the relation between the magnitude of the eigenvalue of a skew symmetric matrix $A$ (which is used in the Cayley map), and the tangent of the half of the relation angle $\theta$.

From the translation parts;

$$
\begin{aligned}
& \frac{v_{1}-a_{12} v_{2}}{1+a_{12}^{2}}=q_{y} \sin \theta+q_{x}(1-\cos \theta) \\
& \frac{a_{12} v_{1}+v_{2}}{1+a_{12}^{2}}=q_{y}(1-\cos \theta)-q_{x} \sin \theta
\end{aligned}
$$

Hence we obtain,

$$
\begin{aligned}
& q_{x}=-\frac{v_{2}}{2 a_{12}} \\
& q_{y}=\frac{v_{1}}{2 a_{12}} .
\end{aligned}
$$

This is the result which gives the center of rotation, $\left(q_{x}, q_{y}\right)$, of the planar motion group $S E(2)$ in terms of the velocity vector $\left(v_{1}, v_{2}\right)$ and $a_{12}$, of the tangent space $s e(2)$.

At this moment we would like to discuss the kinematic meaning of $a_{12}$. If we take a rigid body which moves on a plane (for simplicity let us take xy-plane), like a mobile robot, then the motion of this rigid body only relates with $S E(2)$. So any point with position vector $\overrightarrow{r_{1}}$ on the rigid body rotates to $\overrightarrow{r_{2}}$ around the rotation axis by the angle $\theta$, while the projection $\overrightarrow{r_{1}^{\prime}}$ rotates to $\overrightarrow{r_{2}^{\prime}}$ with the same angle $\theta$ where $\overrightarrow{r_{1}^{\prime}}$ and $\overrightarrow{r_{2}^{\prime}}$ are projections of $\overrightarrow{r_{1}}$ and $\overrightarrow{r_{2}}$ on to the xy-plane respectively.

Now we import a vector $\vec{b}$, which is parallel to the rotation axis (z-axis here) and well known by the Rodrigues vector, to our computations [6]. From Fig.1 it follows that

$$
\tan \left(\frac{\theta}{2}\right)=\frac{\frac{\left|\overrightarrow{r_{2}^{\prime}}-\overrightarrow{r_{1}^{\prime}}\right|}{2}}{\frac{\left|\overrightarrow{r_{1}^{\prime}}+\overrightarrow{r_{2}^{\prime}}\right|}{2}}=\frac{\left|\overrightarrow{r_{2}^{\prime}}-\overrightarrow{r_{1}^{\prime}}\right|}{\left|\overrightarrow{r_{1}^{\prime}}+\overrightarrow{r_{2}^{\prime}}\right|} .
$$

Since the diagonals of the rhombus are perpendicular to each other

$$
\left(\overrightarrow{r_{1}^{\prime}}-\overrightarrow{r_{2}^{\prime}}\right)^{T} \cdot\left(\overrightarrow{r_{1}^{\prime}}+\overrightarrow{r_{2}^{\prime}}\right)=0
$$

where $\overrightarrow{r_{2}^{\prime}}=R \overrightarrow{r_{1}^{\prime}}$. So

$$
\begin{gathered}
\overrightarrow{r_{2}^{\prime}}-\overrightarrow{r_{1}^{\prime}}=R \overrightarrow{r_{1}^{\prime}}-\overrightarrow{r_{1}^{\prime}}=(R-I) \overrightarrow{r_{1}^{\prime}}, \\
\overrightarrow{r_{2}^{\prime}}+\overrightarrow{r_{1}^{\prime}}=(R+I) \overrightarrow{r_{1}^{\prime}},
\end{gathered}
$$




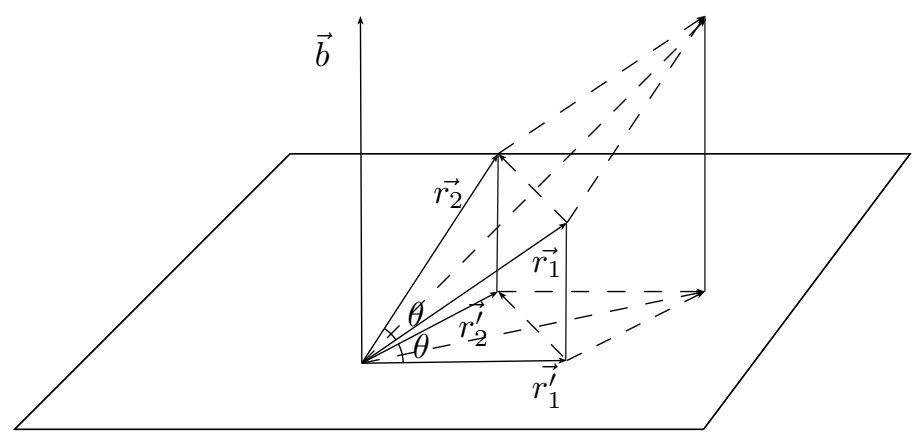

Figure 1. The instantaneous rotation axis for a rigid body and the rhombuses drawn by $\overrightarrow{r_{1}}, \overrightarrow{r_{2}}$ and $\overrightarrow{r_{1}^{\prime}}, \overrightarrow{r_{2}^{\prime}}$.

$$
\begin{gathered}
\overrightarrow{r_{1}^{\prime}}=(R+I)^{-1}\left(\overrightarrow{r_{2}^{\prime}}+\overrightarrow{r_{1}^{\prime}}\right), \\
\left(\overrightarrow{r_{2}^{\prime}}-\overrightarrow{r_{1}^{\prime}}\right)=(R-I)(R+I)^{-1}\left(\overrightarrow{r_{2}^{\prime}}+\overrightarrow{r_{1}^{\prime}}\right) .
\end{gathered}
$$

The matrix $A=(R-I)(R+I)^{-1}$ in (4.4) has the property that for a general vector $\vec{n}$ on xy-plane (it is easy to find $\overrightarrow{r_{1}^{\prime}}$ and $\overrightarrow{r_{2}^{\prime}}$ such that $\left.\left.\overrightarrow{r_{1}^{\prime}}+\overrightarrow{r_{2}^{\prime}}=\vec{n}\right)\right)$. A. $\vec{n}$ is perpendicular to $\vec{n}$ by $(4.3)$.

Hence

$$
\vec{n}^{T} A \vec{n}=\sum_{i, j}\left(a_{i j}+a_{j i}\right) n_{i} n_{j}=0, \quad \forall \vec{n} \in \mathbb{E}^{2},
$$

where $a_{i j}$ are the entries of $A$. So $a_{i j}=-a_{j i}$, that is $A$ is skew symmetric and can be taken as (3.2).

If we extend $A$ to $3 x 3$ matrix space and $\vec{n}$ to $\mathbb{E}^{3}$ by

$A^{\prime}=\left(\begin{array}{ccc}0 & -a_{12} & 0 \\ a_{12} & 0 & 0 \\ 0 & 0 & 0\end{array}\right), n^{\prime}=\left(\begin{array}{c}n_{1} \\ n_{2} \\ 0\end{array}\right)$ then

$$
A^{\prime} \cdot \vec{n}^{\prime}=\vec{b} \times \vec{n}^{\prime}
$$

where $\vec{b}=\left(0,0, a_{12}\right)$ is the Rodrigues vector.

Finally from (4.1), (4.2) and (4.6)

$$
|\vec{b}|=\tan \left(\frac{\theta}{2}\right)=\left|a_{12}\right| .
$$

(4.7) is the relation which combines Rodrigues vector, the exponential map and the Cayley map.

On the other hand

$$
\begin{aligned}
\frac{\overrightarrow{r_{2}^{\prime}}-\overrightarrow{r_{1}^{\prime}}}{\left|\overrightarrow{r_{2}^{\prime}}-\overrightarrow{r_{1}^{\prime}}\right|} & =\vec{s} \times \frac{\overrightarrow{r_{2}^{\prime}}+\overrightarrow{r_{1}^{\prime}}}{\left|\overrightarrow{r_{2}^{\prime}}+\overrightarrow{r_{1}^{\prime}}\right|} \\
\overrightarrow{r_{2}^{\prime}}-\overrightarrow{r_{1}^{\prime}} & =\frac{\left|\overrightarrow{r_{2}^{\prime}}-\overrightarrow{r_{1}^{\prime}}\right|}{\left|\overrightarrow{r_{2}^{\prime}}+\overrightarrow{r_{1}^{\prime}}\right|} \overrightarrow{s^{\prime}} \times\left(\overrightarrow{r_{2}^{\prime}}+\overrightarrow{r_{1}^{\prime}}\right) \\
& =|\vec{b}| \vec{s} \times\left(\overrightarrow{r_{2}^{\prime}}+\overrightarrow{r_{1}^{\prime}}\right) \\
& =\left(\tan \left(\frac{\theta}{2}\right) \vec{s}\right) \times\left(\overrightarrow{r_{1}^{\prime}}+\overrightarrow{r_{2}^{\prime}}\right) \\
& =\left|a_{12}\right| \vec{s} \times\left(\overrightarrow{r_{1}^{\prime}}+\overrightarrow{r_{2}^{\prime}}\right),
\end{aligned}
$$

where $\vec{s}$ is the unit vector along $\vec{b}$ and the equation (4) is the Rodrigues formula for a planar displacement (for the dual form see also [3, 4]). 


\subsection{Application (Three-Link Mechanism)}

Consider the three-link mechanism shown in Fig.2. This planar mechanism has three degrees of freedom to specify the position of a link in a plane. We can use one point $(x, y) \in \mathbb{E}^{2}$ and angle $\theta$ to represent the position and orientation of a link. The joint variables are $\theta_{1}, \theta_{2}, \theta_{3}$, and length of the links are just $q_{1}, q_{2}, q_{3}$. By taking $\theta_{1}=\theta_{2}=\theta_{3}=0$ we define a convenient home configuration. For a final position of manipulator the following rotations are taken;

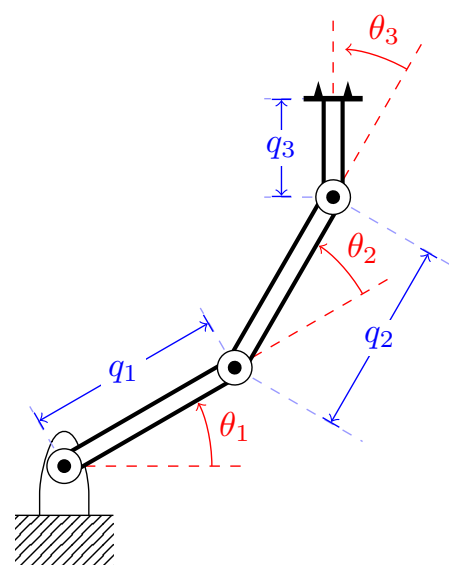

Figure 2. A three-link planar manipulator

First rotate joint 3 by $\theta_{3}$ with center of rotation $\left(\left(q_{1}+q_{2}\right), 0\right)$;

$$
A\left(\theta_{3}\right)=\left(\begin{array}{ccc}
\cos \theta_{3} & -\sin \theta_{3} & \left(q_{1}+q_{2}\right)\left(1-\cos \theta_{3}\right) \\
\sin \theta_{3} & \cos \theta_{3} & -\left(q_{1}+q_{2}\right) \sin \theta_{3} \\
0 & 0 & 1
\end{array}\right)
$$

Rotate joint 2 by $\theta_{2}$ with center of rotation $\left(q_{1}, 0\right)$;

$$
A\left(\theta_{2}\right)=\left(\begin{array}{ccc}
\cos \theta_{2} & -\sin \theta_{2} & q_{1}\left(1-\cos \theta_{2}\right) \\
\sin \theta_{2} & \cos \theta_{2} & -q_{1} \sin \theta_{2} \\
0 & 0 & 1
\end{array}\right)
$$

Finally rotate joint 1 by $\theta_{1}$ with center of rotation $(0,0)$;

$$
A\left(\theta_{1}\right)=\left(\begin{array}{ccc}
\cos \theta_{1} & -\sin \theta_{1} & 0 \\
\sin \theta_{1} & \cos \theta_{1} & 0 \\
0 & 0 & 1
\end{array}\right)
$$

Thus the effect of such a movement is given by the product of the three matrices;

$$
A\left(\theta_{1}\right) A\left(\theta_{2}\right) A\left(\theta_{3}\right)=\left(\begin{array}{ccc}
\cos \left(\theta_{1}+\theta_{2}+\theta_{3}\right) & -\sin \left(\theta_{1}+\theta_{2}+\theta_{3}\right) & l_{x} \\
\sin \left(\theta_{1}+\theta_{2}+\theta_{3}\right) & \cos \left(\theta_{1}+\theta_{2}+\theta_{3}\right) & l_{y} \\
0 & 0 & 1
\end{array}\right)
$$

where the unknown variables $l_{x}$ and $l_{y}$ are given by;

$$
\begin{aligned}
& l_{x}=q_{1} \cos \theta_{1}+q_{2} \cos \left(\theta_{1}+\theta_{2}\right)-\left(q_{1}+q_{2}\right) \cos \left(\theta_{1}+\theta_{2}+\theta_{3}\right) \\
& l_{y}=q_{1} \sin \theta_{1}+q_{2} \sin \left(\theta_{1}+\theta_{2}\right)-\left(q_{1}+q_{2}\right) \sin \left(\theta_{1}+\theta_{2}+\theta_{3}\right) .
\end{aligned}
$$

The forward kinematics of the mechanism can be derived by the product of $A\left(\theta_{1}\right) A\left(\theta_{2}\right) A\left(\theta_{3}\right)$,

$$
\left(\begin{array}{l}
x \\
y \\
1
\end{array}\right)=\left(\begin{array}{ccc}
\cos \left(\theta_{1}+\theta_{2}+\theta_{3}\right) & -\sin \left(\theta_{1}+\theta_{2}+\theta_{3}\right) & l_{x} \\
\sin \left(\theta_{1}+\theta_{2}+\theta_{3}\right) & \cos \left(\theta_{1}+\theta_{2}+\theta_{3}\right) & l_{y} \\
0 & 0 & 1
\end{array}\right)\left(\begin{array}{c}
q_{1}+q_{2}+q_{3} \\
0 \\
1
\end{array}\right) .
$$


Hence we find the kinematic equations;

$$
\begin{aligned}
& x=q_{1} \cos \left(\theta_{1}\right)+q_{2} \cos \left(\theta_{1}+\theta_{2}\right)+q_{3} \cos \left(\theta_{1}+\theta_{2}+\theta_{3}\right) \\
& y=q_{1} \sin \left(\theta_{1}\right)+q_{2} \sin \left(\theta_{1}+\theta_{2}\right)+q_{3} \sin \left(\theta_{1}+\theta_{2}+\theta_{3}\right) \\
& \theta=\theta_{1}+\theta_{2}+\theta_{3} .
\end{aligned}
$$

Since the matrix multiplication $A\left(\theta_{1}\right) A\left(\theta_{2}\right) A\left(\theta_{3}\right)$ is the exponential matrix as the equation (3.1), we can compare the matrix $A\left(\theta_{1}\right) A\left(\theta_{2}\right) A\left(\theta_{3}\right)$ and the Cayley matrix (3). Then

$$
a_{12}=\tan \left(\frac{\left(\theta_{1}+\theta_{2}+\theta_{3}\right)}{2}\right),
$$

as in (4.1), and

$$
\begin{aligned}
& u_{1}=q_{1} \cos \theta_{1}+q_{2} \cos \left(\theta_{1}+\theta_{2}\right)-\left(q_{1}+q_{2}\right) \cos \left(\theta_{1}+\theta_{2}+\theta_{3}\right) \\
& u_{2}=q_{1} \sin \theta_{1}+q_{2} \sin \left(\theta_{1}+\theta_{2}\right)-\left(q_{1}+q_{2}\right) \sin \left(\theta_{1}+\theta_{2}+\theta_{3}\right),
\end{aligned}
$$

as in (4). The equation (4.7) gives the length of the Rodrigues vector and the equation (4.8) yields the position of joints in the plane.

For a 3D-graph of length of the Rodrigues vector and the joint positions of three-link planar mechanism we need two free variables. Taking $\theta_{1}$ constant we have $\theta_{2}, \theta_{3}$ free variables. Let we choose $\theta_{1}=\frac{\pi}{4}, q_{1}=8, q_{2}=4$, $q_{3}=2, \theta_{2}, \theta_{3} \in\left[-\frac{\pi}{2}, \frac{\pi}{4}\right]$. Hence we get

$$
\begin{aligned}
x & =4 \sqrt{2}+4 \cos \left(\frac{\pi}{4}+\theta_{2}\right)+2 \cos \left(\frac{\pi}{4}+\theta_{2}+\theta_{3}\right) \\
y & =4 \sqrt{2}+4 \sin \left(\frac{\pi}{4}+\theta_{2}\right)+2 \sin \left(\frac{\pi}{4}+\theta_{2}+\theta_{3}\right) \\
\theta & =\frac{\pi}{4}+\theta_{2}+\theta_{3} .
\end{aligned}
$$

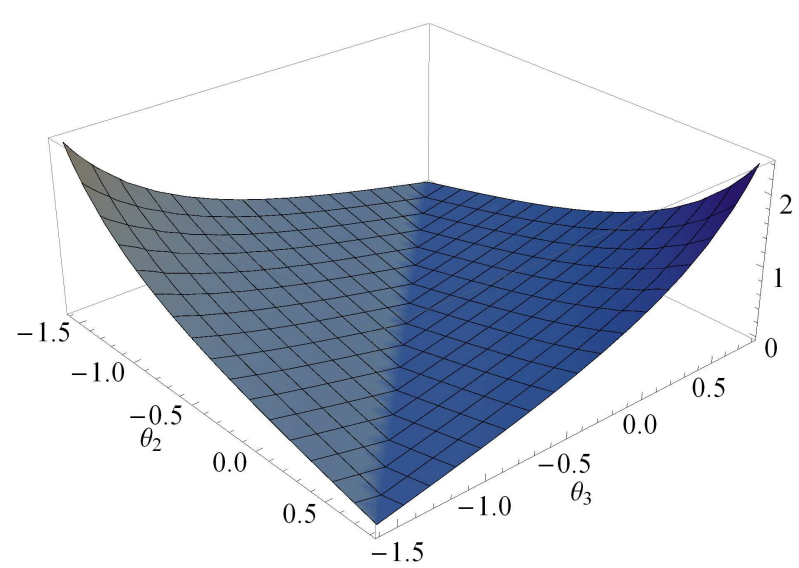

(a) Length of Rodrigues vector

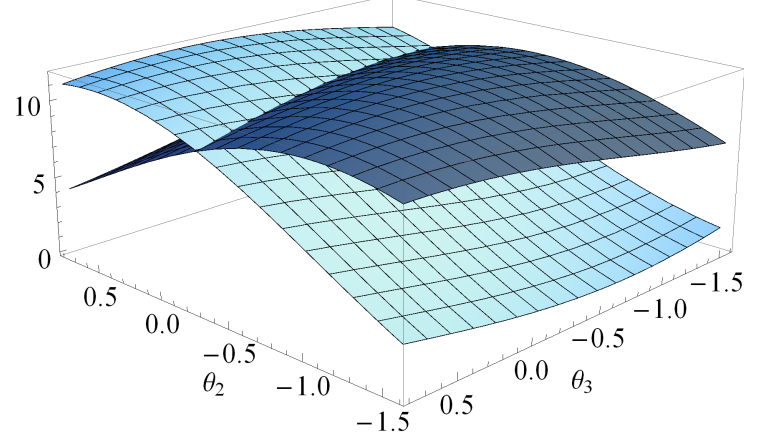

(b) Joint positions

Figure 3. The length of Rodrigues vector and the location of joint positions with $-\frac{\pi}{2} \leq \theta_{2} \leq \frac{\pi}{4}$ (dark) and $-\frac{\pi}{2} \leq \theta_{3} \leq \frac{\pi}{4}$ (light).

Figure 3(a) represents the length of the Rodrigues vector $\vec{b}$ depending on $\theta_{2}$ and $\theta_{3}$, and also figure $3(\mathrm{~b})$ is the joint positions with the same variables, $\theta_{2}$ and $\theta_{3}$. These two figures have information about the eigenvalue of the Lie algebra element $a_{12}$ and the position of the center of rotation, $u_{1}, u_{2}$, in the plane respectively.

\section{Conclusions}

Given a skew symmetric matrix, a Lie algebra element, one can obtain an orthogonal matrix, a Lie group element, in the real n-space[2, 6, 14]. This is known as the Cayley transform. Also it is well known that the 
exponential map is another way to obtain orthogonal matrices from the skew symmetric matrices. So it is natural way to think about a relation between the Cayley map and the exponential map. This is done in $S E(3)$ by Selig [7].

In this study we have found the comparison of the Cayley map and exponential map in $S E(2)$ and also a relation between the eigenvalue of the skew symmetric matrix and the Rodrigues vector is obtained and as an application three joint mechanism is discussed graphically.

\section{Acknowledgments}

We would like to thank the editor and reviewers.

\section{References}

[1] Paraskevopoulos, E., Natsiavas, S.: A new look into the kinematics and dynamics of finite rigid body rotations using Lie group theory . International Journal of Solids and Structures. 50(1), 57-72 (2013).

[2] Müller, H.R.: Sphärische Kinematik. Berlin: Deutscher Verlag der Wissenschaften (1962).

[3] Karakılıç, I.: The Dual Rodrigues Parameters, International Journal of Engineering and Applied Sciences. 2(2), 23-32 (2010).

[4] Karakılıç, I.: Expression of Dual Euler Parameters Using the Dual Rodrigues Parameters and Their Application to the Screw Transformation. Mathematical and Computational Applications, 16(3), 680-689 (2011).

[5] Gallier, J.: Geometric Methods and Applications for Computer Science and Engineering. Springer-Verlag (2000).

[6] McCarthy, J.M.: An Introduction to Theoretical Kinematics. MIT Press (1990).

[7] Selig, J.M.: Cayley maps for SE(3). The International Federation of Theory of Machines and Mechanisms 12th World Congress, Besancon (2007).

[8] Selig, J.M.: Centrodes and Lie Algebra. The International Federation of Theory of Machines and Mechanisms 12th World Congress, Besancon (2007).

[9] Selig, J.M.: Geometric Fundamentals of Robotics. Ed:Gries D., Schneider T.R., Second Edition, Springer-Verlag, London (2005).

[10] Selig, J.M.: Introductory Robotics. Prentice Hall International Ltd, UK (1992).

[11] Selig, J.M.: Lie Groups and Lie Algebras in Robotics. Lecture Notes. South Bank University London SE1 0AA, U.K., 101-125 (2006).

[12] Selig, J.M.: On the Geometry of Point-Plane Constraints on Rigid-Body Displacements. Acta Applicandae Mathematicae. 116(2), 133-155 (2011).

[13] Overfeli, M., Kumar, V., Harwin, W.S.: Methods for Kinematic Modeling of Biological and Robotic Systems. Medical Engineering and Physics 22, 509-520 (2000).

[14] Bottema, O., Roth, B.: Theoretical Kinematics. North-Holland Publishing, Amsterdam. Reprinted by Dover, New York (1990).

[15] Murray, R.M., Li, Z., Sastry, S.S.: A Mathematical Introduction to Robotic Manipulation. CRC Press, Boca Raton (1994).

[16] Ebetiuc, S., Staab, H.: Applying Differential Geometry to Kinematic Modelling in Mobile Robotics. WSEAS Int. Conf. On Dynamical Systems and Control, Venice, Italy, 106-112 (2005).

\section{Affiliations}

İLHAN KARAKILIÇ

AdDress: Department of Mathematics, Dokuz Eylül University, Fen Fakültesi, Tinaztepe Kampüsü, 35390 Buca, İzmir-Turkey.

E-MAIL: ilhan.karakilic@gmail.com

ORCID ID:0000 000187184268

\section{SONER ERKUŞ}

ADDRESs: Department of Mathematics, Dokuz Eylül University, Fen Fakültesi, Tinaztepe Kampüsü, 35390 Buca, İzmir-Turkey.

E-MAIL: sonererkus@gmail.com

ORCID ID:0000 000346598629 